\title{
Investigating the Effect of Reaction Time on Carbon Dot Formation, Structure, and Optical Properties
}

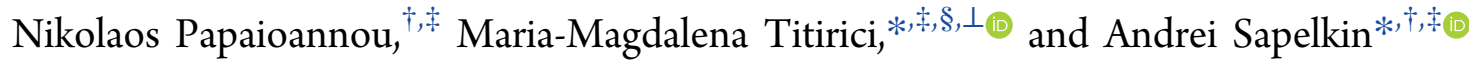 \\ ${ }^{\dagger}$ School of Physics and Astronomy, Queen Mary University of London, 327 Mile End Road, London E1 4NS, U.K. \\ ${ }^{\ddagger}$ Materials Research Institute and ${ }^{\S}$ School of Engineering and Materials Science, Queen Mary University of London, Mile End Road, \\ E1 4NS London, U.K. \\ ${ }^{\perp}$ Department of Chemical Engineering, Imperial College London, South Kensington Campus, SW7 2AZ, UK
}

\section{Supporting Information}

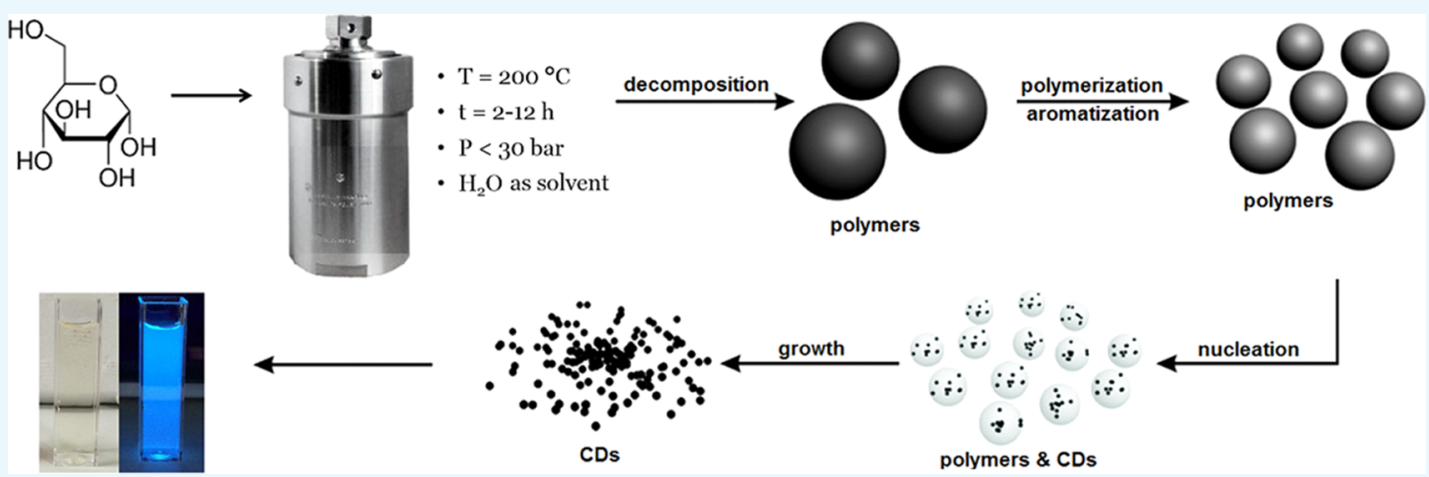

ABSTRACT: Carbon dots, a young member of the carbon nanomaterial family, are quasi-spherical nanoparticles, which have fluorescent properties as their key characteristic. A wide range of starting materials and synthetic routes have been reported in the literature, divided into two main categories: a top-down and bottom-up approach. Moreover, a series of different parameters that affect the properties of carbon dots have been investigated, including temperature, starting $\mathrm{pH}$, as well as precursor concentration. However, the effect of reaction time has not been extensively monitored. In our study, a biomass derivative was treated hydrothermally with varying reaction times to draw a solid formation mechanism. In addition, we monitored the effect of reaction time on optical and structural characteristics, as well as the chemical composition of our materials. Our key findings include a four-stage formation mechanism, a higher level of crystallinity, and an increasing brightness over reaction time.

\section{INTRODUCTION}

Carbon dots (CDs) are a new class of nanomaterials, which have attracted tremendous attention due to their unique photoluminescent (PL) properties, biocompatibility, and low toxicity. ${ }^{1,2}$ Thus, they appear as rising candidates to replace the metal-based quantum dots in various applications such as bioimaging $^{3-5}$ and biosensing, ${ }^{6,7}$ drug delivery, ${ }^{8}$ and photocatalysis. ${ }^{9}$ CDs are quasi-spherical particles with size of a few nanometer, and among their key characteristics are hydrophilicity, resistance against photobleaching, and facile surface modification. ${ }^{10,11}$ They were first reported in 2004 when Xu et al. accidentally discovered fluorescent particles during the purification process of single-walled carbon nanotubes produced by arc discharge. ${ }^{12}$ Since then, a new field triggered with studies focused on their fluorescent properties.

The synthesis of CDs can be classified according to their synthetic methods into two main strategies: top-down and bottom-up. Top-down strategy refers to the breaking down of large-sized carbonaceous materials by laser ablation, ${ }^{13}$ arc discharge, ${ }^{12}$ acidic oxidation, ${ }^{14}$ or electrochemical approaches. $^{15}$ The bottom-up strategy refers to synthetic approaches from molecular precursors through thermal or combustion treatments, ${ }^{2,16,17}$ microwave synthetic routes, ${ }^{18,19}$ and other solution-based synthetic methods. ${ }^{20,21}$ Precursors such as saccharides, ${ }^{22}$ amino acids, ${ }^{23}$ and biopolymers ${ }^{17}$ are among the most common in this strategy. Structural and optical characteristics of CDs have gained vast attention in the research community since their first discovery in $2004 .^{12,24,25}$ Many parameters have been examined including reaction temperature, ${ }^{26-29}$ precursor concentration, ${ }^{30}$ the addition of any doping elements, ${ }^{31-34}$ and also the ratio between the starting materials. ${ }^{2,35}$ However, there are only a few published reports assessing the effect of the reaction time to monitor how their properties evolve. ${ }^{30,36,37}$

In this report, the experimental strategy involved is hydrothermal carbonization (HTC) to convert the biomass derivatives, such as glucose, into carbonaceous materials with water as a solvent at mild conditions $\left(200{ }^{\circ} \mathrm{C}\right.$ and 30 bars of

Received: June 17, 2019

Accepted: September 16, 2019

Published: December 12, 2019 

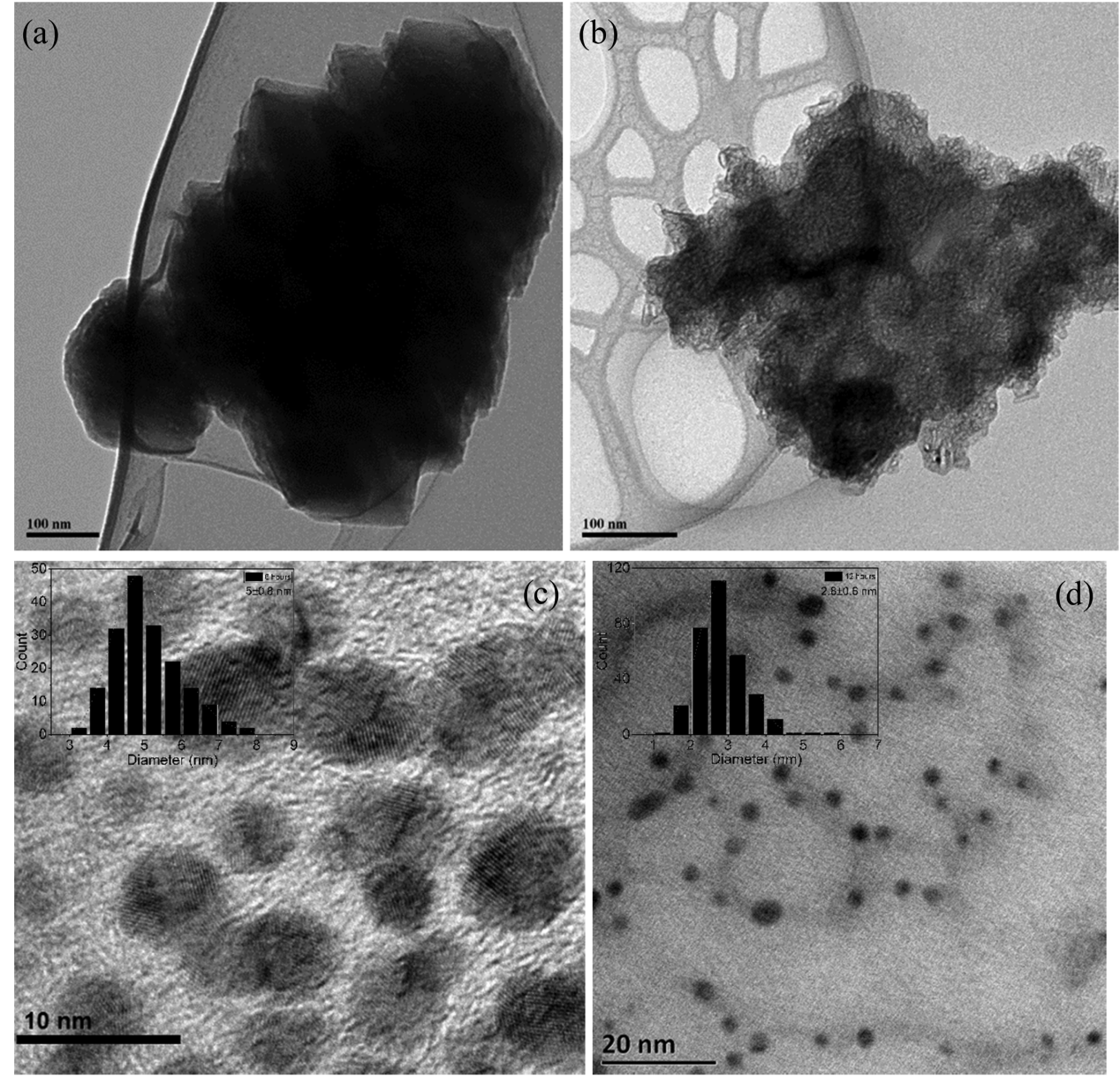

Figure 1. TEM micrographs and size distribution charts (inserts) of CDs synthesized after (a) $2 \mathrm{~h},(\mathrm{~b}) 4 \mathrm{~h}$, (c) $6 \mathrm{~h}$, and (d) $12 \mathrm{~h}$.
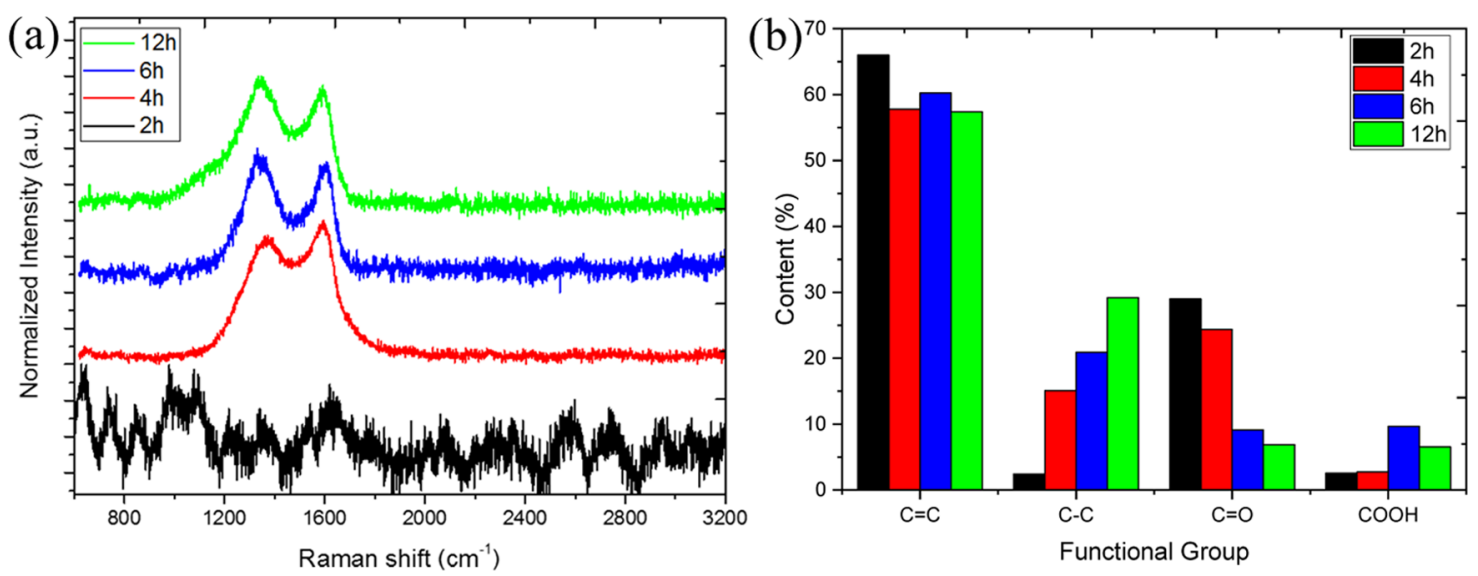

Figure 2. (a) Raman spectra (for comparison, Raman spectra of the precursor are shown in Figure S2) and (b) summary of X-ray photoelectron spectroscopy (XPS) data analysis of CDs.

self-generated pressure) and varying the reaction time from 2 to $12 \mathrm{~h}$. This strategy was selected to get the maximum information possible about how these materials form and what affects their optical and structural properties, as well as their chemical composition using a combination of structural and optical characterization methods. Briefly, we identified a fourstage formation mechanism, which leads to smaller particle size CDs with a higher level of crystallinity.

\section{RESULTS AND DISCUSSION}

In Figure 1, transmission electron microscopy (TEM) micrographs of CDs with synthesis times of $2,4,6$, and $12 \mathrm{~h}$ are presented. After $2 \mathrm{~h}$ of the synthesis process (Figure 1a), no nanometer-sized particles were observed and only flake-like structures can be seen. Varying sizes of these structures were observed from $400 \mathrm{~nm}$ to as high as a few micrometers. In Figure $1 \mathrm{~b}$, images of the sample after a $4 \mathrm{~h}$ synthesis are shown. In this case, there are still ill-shaped particles with sizes of around $200-300 \mathrm{~nm}$, which are significantly smaller compared 
Scheme 1. Potential Formation Mechanism of CDs Hydrothermally Synthesized from Glucose
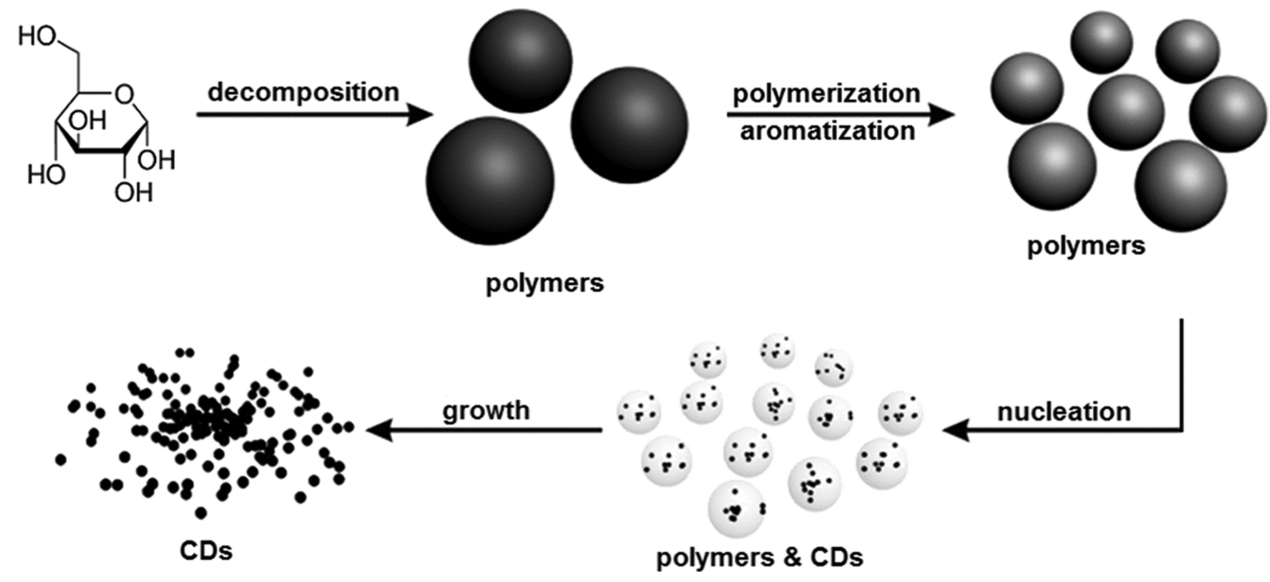

to the previous sample but not representative of the carbon dots suggesting aggregated and unreacted precursor. Figure 1c illustrates the sample produced after $6 \mathrm{~h}$ of hydrothermal treatment where a clear transition to nanometer-sized, quasispherical particles occurred, with an average particle size of 5 $\mathrm{nm}$. More detailed information on the atomic structure of these particles was thoroughly presented in a previous report. ${ }^{38}$ In summary, a diverse structure of this sample was shown to include carbon-onion type, amorphous carbon, and graphiticlike carbon structures. Finally, in Figure 1d, TEM data for CDs after $12 \mathrm{~h}$ of HTC are shown. Size of the quasi-spherical particles here is further reduced, averaging at $2.8 \mathrm{~nm}$. Moreover, this sample showed crystalline planes with $2.06 \AA$ separation (Figure S1), identified as the (111) planes of diamond $^{39,40}$ and the (100) planes of graphite. ${ }^{41,42}$ Additionally, in Figure 1d, one can observe strips extending from particle to particle, attached to the spherical cores. At this stage, the origin of these strips remains unclear and requires further investigation. A preliminary interpretation at this stage is that these strips are amorphous (compared to the crystalline core of the CDs) and similar to those observed in the report by $\mathrm{Xu}$ et al., ${ }^{43}$ who confirmed the presence of amorphous $\mathrm{sp}^{3}$ surface regions in $\mathrm{CDs}$ along with the crystalline $\mathrm{sp}^{2}$ carbon core. Our assumption is that the carbon dots are "crystallized" from the amorphous carbon stripes/cones. We are hoping to confirm this in upcoming small-angle X-ray scattering experiments performed "in situ" during the hydrothermal process. Overall, the particle size reduced over reaction time, while samples appeared more crystalline. The latter is further supported by a recent study reported in the literature. ${ }^{44}$

To obtain further information on how reaction time affects the structural characteristics of our samples, we utilized Raman scattering as it can provide a wealth of information in carbon systems. $^{38,45,46}$ In particular, the Raman data of CDs in Figure $2 \mathrm{a}$ indicate that samples have a significant degree of disorder, which is reflected in an increased $D$ and $G$ peak width. ${ }^{46} \mathrm{D}$ band originates from aromatic conjugated $\mathrm{sp}^{2}$ 6-membered carbon rings; ${ }^{47}$ hence, an increase in $\mathrm{D}$ peak intensity reflects a large number of such ring clusters. Consequently, broadening of the $\mathrm{D}$ band reflects a disorder in aromatic clusters. The position of the $\mathrm{D}$ band strongly depends on excitation energy, ${ }^{48}$ and for $\mathrm{He}-\mathrm{Ne}(632.8 \mathrm{~nm})$ laser used here, the peak is expected at around $1325 \mathrm{~cm}^{-1}$ in graphite. The observed $\mathrm{G}$ bands are clearly asymmetric, which, on the lower wavelength side of the peak in nanoscale systems, is usually associated with the phonon confinement, ${ }^{49}$ thus suggesting that the particle size is sufficiently small for bulk phonon dispersion description to breakdown. As shown in Table S1, G band full width at half-maximum is decreasing as a function of synthesis time, which comes to an agreement with the TEM analysis, thus suggesting a better ordering of carbon rings. On the contrary, the peak width of the $\mathrm{G}$ band and $I_{\mathrm{D}} / I_{\mathrm{G}}$ decreases during the same period suggesting a drop in average size, which is again consistent with the TEM analysis.

$\mathrm{X}$-ray photoelectron spectroscopy (XPS) findings show two typical peaks, $\mathrm{C} 1 \mathrm{~s}$ at $284.8 \mathrm{eV}$ and $\mathrm{O} 1 \mathrm{~s}$ at $531.8 \mathrm{eV}$. In the high-resolution spectra (Figure S3), C 1s band was deconvoluted into four peaks, which correspond to $\mathrm{sp}^{2}$ carbons $(\mathrm{C}=\mathrm{C}, 284.8 \mathrm{eV}), \mathrm{sp}^{3}$ carbons $(\mathrm{C}-\mathrm{C}, 286.1 \mathrm{eV})$, carbonyl carbons $(\mathrm{C}=\mathrm{O}, 287.8 \mathrm{eV})$, and carboxyl carbons $(\mathrm{COOH}$, $289 \mathrm{eV}){ }^{44,50,51}$ The $\mathrm{O} 1 \mathrm{~s}$ band contains two peaks at 531.8 and $533.2 \mathrm{eV}$ for $\mathrm{C}=\mathrm{O}$ and $\mathrm{C}-\mathrm{O}$, respectively. ${ }^{50}$ The XPS intensity at $286.1 \mathrm{eV}$ gradually increases from a glucose- $2 \mathrm{~h}$ sample to glucose- $12 \mathrm{~h}$ sample, indicating a corresponding increase in the content of $\mathrm{sp}^{3}$ carbons in the CDs as analysis from the $C 1$ s spectra shown in Figure $2 b$. XPS results showed a higher level of homogeneity in the chemical composition of our CDs and that further correlate well with the diffraction patterns and Raman data, which showed an increasing degree of crystallization over reaction time. Overall, information extracted from the above table suggests the presence of the graphitic structures, which is confirmed by the $\mathrm{sp}^{2} / \mathrm{sp}^{3}$ carbon fraction observed in the $\mathrm{C} 1 \mathrm{~s}$ peak for all samples. $\mathrm{C} 1 \mathrm{~s}$ peaks appear narrower over time, further indicating accumulation in bonding energies suggesting an increasing homogeneity in CDs composition.

Based on these data, we propose the following mechanism (illustrated in Scheme 1) of the evolution of precursors during synthesis. Initially, dehydration of the precursor occurs, which leads to aggregation of decomposed products and mild condensation that forms large-sized polymer nanoparticles (see Figure 1a). These polymer nanoparticles shrink due to continuous intramolecular dehydration as heating proceeds. At this stage, $\mathrm{C}-\mathrm{C}$ bonds are formed, and at the same time, aromatic clusters are shaped inside the polymers. Once the concentration of so-formed clusters reaches the critical supersaturation point, the nucleation of CDs takes place. ${ }^{52}$ (Also see Figure 1d.) During this stage, nuclei are formed by the diffusion of the aromatic clusters toward the particle surface, and simultaneous passivation with various functional 

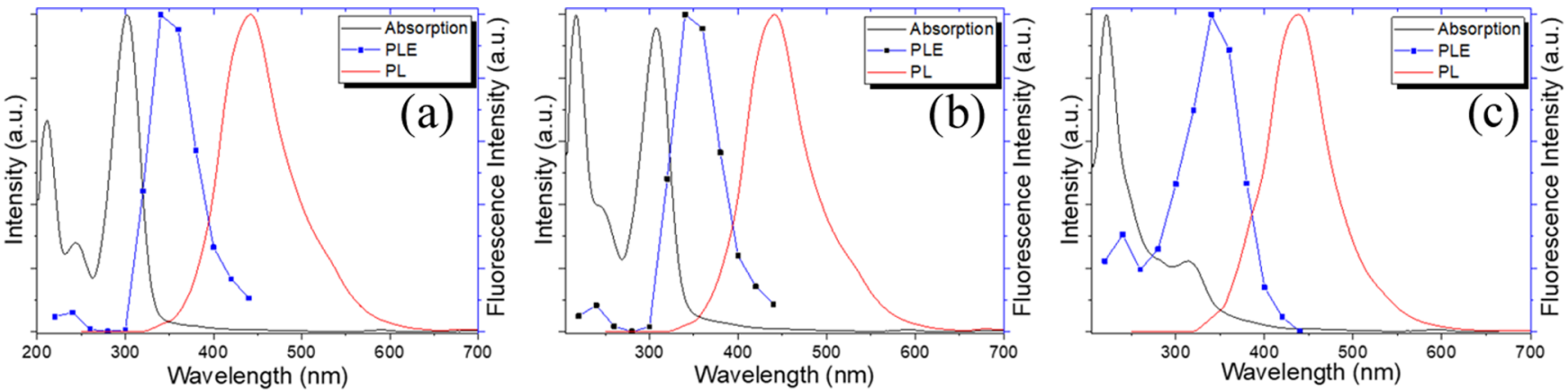

Figure 3. Normalized absorption, PLE, and PL emission plots of (a) 4 h, (b) 6 h, and (c) 12 h CDs.
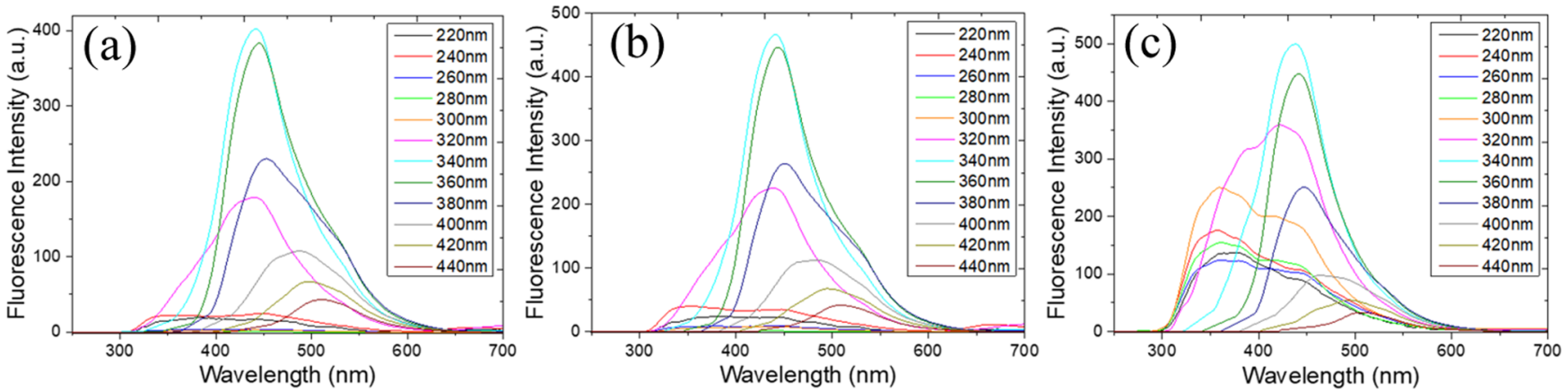

Figure 4. PL spectra of (a) 4 h, (b) 6 h, and (c) 12 h CDs.

groups (hydroxyl, carbonyl, carboxyl, etc.) takes place. Over time, polymer nanoparticles tend to extinct (convert) giving place to $\mathrm{CDs}$; therefore, the ratio polymers:dots decrease leading to a smaller particle size of CDs and less polycrystallinity (Figure 1d). In the last stage, the smaller particle size and narrower distribution are narrower as a result of polymer decomposition.

Further, origins of light absorption and emission will be investigated. The variation in surface functionality could be identified with a change in UV-vis absorption spectra. ${ }^{53}$ In this manner, UV-vis spectra of as-synthesized CDs are shown in Figure 3, where a clear effect of reaction time is observed. The $4 \mathrm{~h}$ synthesis sample showed two peaks, one at $244 \mathrm{~nm}$ $(5.08 \mathrm{eV})$, corresponding to the $\pi \rightarrow \pi^{*}$ transition of the aromatic $\mathrm{sp}^{2}$ domains, ${ }^{54}$ and another strong absorption band at $303 \mathrm{~nm}(4.09 \mathrm{eV})$, usually present in molecular-like energy states and attributed to the $\mathrm{n} \rightarrow \pi^{*}$ transition of $\mathrm{C}=\mathrm{O}$ bond. ${ }^{26,37,55}$ The later adsorption band was maintained in the sample produced after $6 \mathrm{~h}$, while the $244 \mathrm{~nm}$ was replaced by an absorption shoulder. Finally, at $12 \mathrm{~h}$, the previously sharp peak is weakened, whereas a new one was formed at $\sim 320 \mathrm{~nm}$ $(3.87 \mathrm{eV})$, which is assigned to $\mathrm{n} \rightarrow \pi^{*}$ transitions by nonbonding orbitals such as $\mathrm{C}=\mathrm{O}$ bonds. ${ }^{56,57}$ While absorption measurements provide information about transitions between the ground and the excited states, photoluminescence excitation (PLE) provides information about the energy levels contributing to the specific light emission bands. The normalized absorption, PLE, and PL spectra of CDs are presented in Figure 3. CD spectra shown in Figure 3 exhibit two PLE bands at $240 \mathrm{~nm}(5.17 \mathrm{eV})$ and $340 \mathrm{~nm}(3.65 \mathrm{eV})$, which are attributed to the presence of different types of emission sites on their surface. ${ }^{58}$

$\mathrm{PL}$ emission in each case is located in the blue region of the electromagnetic spectrum (at $\sim 440 \mathrm{~nm}$ ). From the obtained PLE data, one can see that there is one dominant excitation peak at $340 \mathrm{~nm}$, which indicates that the blue emission of CDs originates from energy levels located in the range of electronic transition on $\mathrm{C}=\mathrm{O}$ bonds. ${ }^{59}$ Most likely, they correspond to highest occupied molecular orbital-lowest unoccupied molecular orbital (HOMO-LUMO) energy levels (e.g., and $\pi-\pi^{*}$ transitions) in $\mathrm{sp}^{2}$ bound carbon clusters.

Emission spectra were recorded using excitation wavelengths ranging from 220 to $480 \mathrm{~nm}$ with a step of $20 \mathrm{~nm}$. The detailed excitation-dependent PL experiments reveal a complex picture of light emission with strong emission in the blue-green region of the visible light spectrum, as presented in Figure 4. CDs synthesized after $2 \mathrm{~h}$ did not show any PL; therefore, they were excluded from this analysis. Despite the complex structure of PL spectra suggesting multiple sources of emission, one can broadly identify two distinct regions common for all samples, clearly pointing to a discrete transition between different emission channels as the excitation wavelength is changed. One is upon excitation between 200 and $340 \mathrm{~nm}$, where PL peak positions are nearly independent of the excitation and another is upon excitation between 360 and $480 \mathrm{~nm}$, where PL peak positions are excitation-dependent. A number of published reports show CDs with similar PL behavior with two emission channels. ${ }^{26,60-63}$

Nevertheless, light emission occurred from samples synthesized after $4 \mathrm{~h}$ is unusual, given that they showed large polymeric aggregates in TEM (Figure 1b). Fluorescence, in this case, could possibly be ascribed to a combination of molecular fluorophores, which form during the early stages of the synthesis. Such molecules are either bound to the particle surface or embedded in the amorphous matrix. As reaction time increases, they give way to the growth of aromatic domains comprising carbon and/or nitrogen atoms. This agrees well with the chemical composition analysis and particularly the XPS results (Figure 2b), where the 
contribution of $\mathrm{sp}^{3}$-hybridized carbons increases with reaction time.

Photoluminescent quantum yield (PLQY) is an accurate index revealing information on the efficiency of fluorescent materials. The most consistent method for calculating PLQY is the comparative method of Williams et al. ${ }^{64}$ The PLQY of CDs is illustrated in Figure 5, where values increase linearly over reaction time.

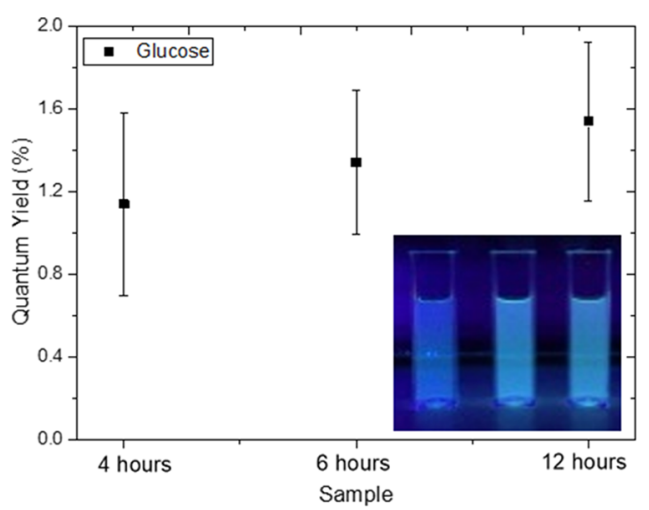

Figure 5. PLQY values of CDs. The inset shows the picture of our samples under a UV lamp excitation. Photograph courtesy of Nikolaos Papaioannou.

The radiative lifetime of emission is an important characteristic of light-emitting nanoparticles, as different radiative lifetimes may correspond to different light emission mechanisms. To further explore the origin of light emission of our CD samples, time-resolved PL decay spectra were collected. Employing excitation wavelengths from both excitationindependent and excitation-dependent emission regions and an emission range between 440 and $640 \mathrm{~nm}$, time-resolved PL decay spectra were recorded. Example decay traces of CDs along with their fitting curves are presented in Figure S4. We found that the best fit to the data required using biexponential functions. The biexponential behavior of CDs indicates that two different emissive sites are present and the fluorescence state is presumably due to the carbon core and surface traps. ${ }^{65,66}$ Full studies were further performed, and the average PL lifetimes extracted from the time-resolved PL decays were compared and are presented in Figure 6. The calculated lifetime values showed different evolutions at short and long excitation wavelengths. At short excitation wavelengths, CDs showed gradually shorter lifetimes upon reaction time increase, obtaining maximum values of 7, 6.5, and $6 \mathrm{~ns}$ after 4,6 , and 12 h syntheses, respectively. When excited at longer wavelengths, glucose- $4 \mathrm{~h}$ and glucose- $6 \mathrm{~h}$ samples showed similar profile with lifetimes shorter compared to the previous excitation region, which were gradually decreasing over emission wavelength from $\sim 6 \mathrm{~ns}$ down to $4 \mathrm{~ns}$. On the contrary, lifetimes of glucose-12 h sample appeared significantly longer, but again had a decreasing trend, implying that a different PL mechanism controls its fluorescence.

The evolution of the time-resolved PL indicates that two different pathways of light emission are responsible for the fast (long-wavelength emissions) and the slow (short-wavelength emissions) decays, respectively. Contrary to previous studies, ${ }^{67}$ the obtained lifetimes (apart from glucose-12 h sample) do not obey the established mechanism, where the average lifetime progressively lengthens with increasing emission wavelengths.

Overall, when structural and optical data were considered collectively, they indicate HOMO-LUMO transitions within the carbon core as the origin of the excitation-independent PL light emission present at short-wavelength excitations, while excitation-dependent PL emission present at longer wavelength excitations is most likely due to the surface defect states, as illustrated in Scheme S1. Taking into account the surfacerelated analysis, the origin of light emission is attributed to the interaction between the carbon core and the surface functional groups (hydroxyl, carboxyl, carbonyl, amines, and amides) upon light absorption.

\section{CONCLUSIONS}

In this study, CDs were synthesized hydrothermally from glucose with varying reaction time from 2 to $12 \mathrm{~h}$. TEM showed a decrease in particle size, while a higher level of monodispersity over reaction time was attained. Based on the above, the formation mechanism of $\mathrm{CD}$ particles was proposed, suggesting four stages including precursor decomposition, followed by polymerization/aromatization, nucleation, and growth. High-resolution TEM (HRTEM) and diffraction patterns showed that less crystalline phases were present at longer reaction times. Finally, HRTEM findings suggested that CDs are possibly composed of a graphitic $\mathrm{sp}^{2}$ carbon core functionalized with peripheral carboxylic/carbonyl groups and $\mathrm{sp}^{3}$ carbon defects. Further, results from Raman spectroscopy indicated that although samples possessed a significant degree of disorder, they showed a higher level of crystallinity as reaction time increased. That imprints on the decreasing $I_{\mathrm{D}} / I_{\mathrm{G}}$ ratio and peak width of the $\mathrm{G}$ band, which was in good agreement with the TEM findings. As per optical properties, PL emission spectra revealed two distinct regions
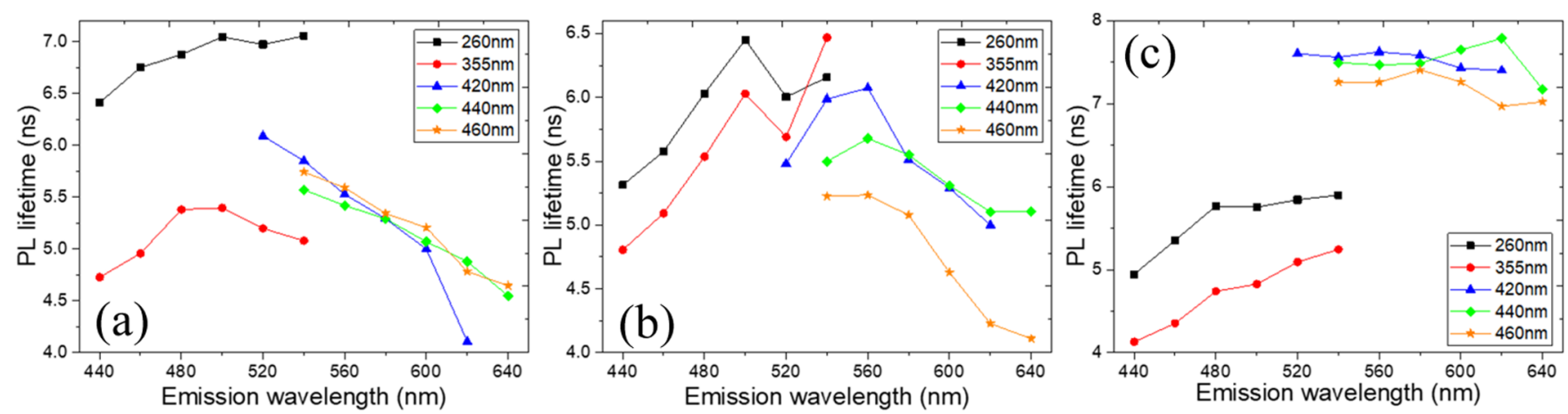

Figure 6. Average PL lifetimes under various excitation wavelengths over the whole emission range of (a) $4 \mathrm{~h},(\mathrm{~b}) 6 \mathrm{~h}$, and (c) $12 \mathrm{~h} \mathrm{CDs}$. 
common for all samples pointing to a discrete transition between different emission channels as the excitation wavelength is changed. Finally, time-resolved PL measurements proposed that CDs show two different pathways of light emission: the fast (long-wavelength emissions) and the slow (short-wavelength emissions) decays.

\section{MATERIALS AND METHODS}

D-(+)-Glucose $(\geq 99.5 \%)$, quinine sulfate dihydrate, and sulfuric acid (reagent grade, 95-98\%) were purchased from Sigma-Aldrich. Glucose was used as a precursor for the hydrothermal synthesis of CDs. Aqueous solution ( $4 \% \mathrm{w} / \mathrm{v})$ of the precursor was prepared and placed in a Teflon-lined, stainless steel autoclave, which underwent hydrothermal treatment at $200{ }^{\circ} \mathrm{C}$ with variable reaction time $(2,4,6$, and $12 \mathrm{~h}$ ). The obtained orange to dark brown liquid phase was centrifuged at $20000 \mathrm{rpm}$ for $10 \mathrm{~min}$ to separate the liquid containing fluorescent $\mathrm{CDs}$ from the solid black precipitate. The liquid phase containing CDs were then filtered using standard syringe filters and further membrane dialyzed against water and freeze-dried for further characterization. TEM was performed on a JEOL JEM-2010 electron microscope. Raman spectra were obtained through a Renishaw 1000 microspectrometer. XPS measurements were carried out on a KAlpha spectrometer utilizing a monochromatic Al K $\alpha$ X-ray source. UV-vis absorption spectra were recorded with a Perkin Elmer Lambda LS35 UV-vis spectrometer. PL spectra were recorded with a Perkin Elmer LS55 fluorescence spectrometer. For the time-resolved PL measurements, laser light was generated by a Continuum Surelite (SLI-10) laser and the beam then passes through an optical parametric oscillator. Then, photoluminescence was collected and collimated onto a Jobin Yvon Horiba Triax 550 spectrometer. A liquid nitrogen cooled photomultiplier tube (PMT) was used to detect and multiply the signal collected from the spectrometer. The response from the PMT was then sent to an oscilloscope and finally the PL spectra and a computer recorded lifetime data. PLQY $(\Phi)$ was calculated by comparing CDs to the reference quinine sulfate $(\Phi=0.54)$. By plotting the integrated fluorescence intensity vs absorbance values for both CDs and quinine sulfate, the slope of the curves was determined and the PLQY of CDs was calculated. ${ }^{64,68}$

\section{ASSOCIATED CONTENT}

\section{S Supporting Information}

The Supporting Information is available free of charge at https://pubs.acs.org/doi/10.1021/acsomega.9b01798.

Electron diffraction pattern, Raman peak fitting analysis results, Raman spectra of glucose precursor, detailed XPS spectra over reaction time and decay traces, accompanied by their fitting curves of CDs; finally, a proposed schematic approach of PL mechanism of CDs (PDF)

\section{AUTHOR INFORMATION}

\section{Corresponding Authors}

*E-mail: m.m.titirici@qmul.ac.uk (M.-M.T.).

*E-mail: a.sapelkin@qmul.ac.uk (A.S.).

ORCID $\odot$

Maria-Magdalena Titirici: 0000-0003-0773-2100

Andrei Sapelkin: 0000-0001-7648-8215

\section{Notes}

The authors declare no competing financial interest.

\section{ACKNOWLEDGMENTS}

The authors gratefully acknowledge Prof. W. P. Gilling for acces to the PL lifetime measurement facility and funding by the Materials Research Institute. XPS spectra were obtained at the National EPSRC XPS Users' Service (NEXUS) at Newcastle University, an EPSRC Mid-Range Facility. This work is supported by the EPSRC grant number EP/R021554/ 1.

\section{REFERENCES}

(1) Georgakilas, V.; Perman, J. A.; Tucek, J.; Zboril, R. Broad family of carbon nanoallotropes: classification, chemistry, and applications of fullerenes, carbon dots, nanotubes, graphene, nanodiamonds, and combined superstructures. Chem. Rev. 2015, 115, 4744-4822.

(2) Zhu, S.; Meng, Q.; Wang, L.; Zhang, J.; Song, Y.; Jin, H.; Zhang, K.; Sun, H.; Wang, H.; Yang, B. Highly photoluminescent carbon dots for multicolor patterning, sensors, and bioimaging. Angew. Chem., Int. Ed. 2013, 52, 3953-7.

(3) Loukanov, A.; Sekiya, R.; Yoshikawa, M.; Kobayashi, N.; Moriyasu, Y.; Nakabayashi, S. Photosensitizer-Conjugated Ultrasmall Carbon Nanodots as Multifunctional Fluorescent Probes for Bioimaging. J. Phys. Chem. C 2016, 120, 15867-15874.

(4) Wang, Z.; Fu, B.; Zou, S.; Duan, B.; Chang, C.; Yang, B.; Zhou, X.; Zhang, L. Facile construction of carbon dots via acid catalytic hydrothermal method and their application for target imaging of cancer cells. Nano Res. 2016, 9, 214-223.

(5) Kim, S.; Choi, Y.; Park, G.; Won, C.; Park, Y.-J.; Lee, Y.; Kim, B.S.; Min, D.-H. Highly efficient gene silencing and bioimaging based on fluorescent carbon dots in vitro and in vivo. Nano Res. 2017, 503.

(6) Choudhary, R.; Patra, S.; Madhuri, R.; Sharma, P. K. EquipmentFree, Single-Step, Rapid, "On-Site" Kit for Visual Detection of Lead Ions in Soil, Water, Bacteria, Live Cells, and Solid Fruits Using Fluorescent Cube-Shaped Nitrogen-Doped Carbon Dots. ACS Sustainable Chem. Eng. 2016, 4, 5606-5617.

(7) Gao, X.; Lu, Y.; Zhang, R.; He, S.; Ju, J.; Liu, M.; Li, L.; Chen, $\mathrm{W}$. One-pot synthesis of carbon nanodots for fluorescence turn-on detection of $\mathrm{Ag}+$ based on the $\mathrm{Ag}+$-induced enhancement of fluorescence. J. Mater. Chem. C 2015, 3, 2302-2309.

(8) Wu, Y. F.; Wu, H. C.; Kuan, C. H.; Lin, C. J.; Wang, L. W.; Chang, C. W.; Wang, T. W. Multi-functionalized carbon dots as theranostic nanoagent for gene delivery in lung cancer therapy. Sci. Rep. 2016, 6, No. 21170.

(9) Liu, J.; Liu, Y.; Liu, N.; Han, Y.; Zhang, X.; Huang, H.; Lifshitz, Y.; Lee, S. T.; Zhong, J.; Kang, Z. Water splitting. Metal-free efficient photocatalyst for stable visible water splitting via a two-electron pathway. Science 2015, 347, 970-974.

(10) Li, H.; Kang, Z.; Liu, Y.; Lee, S.-T. Carbon nanodots: synthesis, properties and applications. J. Mater. Chem. 2012, 22, 24230-24253.

(11) Baker, S. N.; Baker, G. A. Luminescent carbon nanodots: emergent nanolights. Angew. Chem., Int. Ed. 2010, 49, 6726-6744.

(12) Xu, X.; Ray, R.; Gu, Y.; Ploehn, H. J.; Gearheart, L.; Raker, K.; Scrivens, W. A. Electrophoretic analysis and purification of fluorescent single-walled carbon nanotube fragments. J. Am. Chem. Soc. 2004, 126, 12736-12737.

(13) Sun, Y. P.; Zhou, B.; Lin, Y.; Wang, W.; Fernando, K. A.; Pathak, P.; Meziani, M. J.; Harruff, B. A.; Wang, X.; Wang, H.; Luo, P. G.; Yang, H.; Kose, M. E.; Chen, B.; Veca, L. M.; Xie, S. Y. Quantumsized carbon dots for bright and colorful photoluminescence. J. Am. Chem. Soc. 2006, 128, 7756-7757.

(14) Sun, D.; Ban, R.; Zhang, P.-H.; Wu, G.-H.; Zhang, J.-R.; Zhu, J.J. Hair fiber as a precursor for synthesizing of sulfur- and nitrogen-codoped carbon dots with tunable luminescence properties. Carbon 2013, 64, 424-434.

(15) Zhou, J.; Booker, C.; Li, R.; Zhou, X.; Sham, T. K.; Sun, X.; Ding, Z. An electrochemical avenue to blue luminescent nanocrystals 
from multiwalled carbon nanotubes (MWCNTs). J. Am. Chem. Soc. 2007, 129, 744-745.

(16) Wang, J.; Peng, F.; Lu, Y.; Zhong, Y.; Wang, S.; Xu, M.; Ji, X.; $\mathrm{Su}$, Y.; Liao, L.; He, Y. Large-Scale Green Synthesis of Fluorescent Carbon Nanodots and Their Use in Optics Applications. Adv. Opt. Mater. 2015, 3, 103-111.

(17) Yang, Y.; Cui, J.; Zheng, M.; Hu, C.; Tan, S.; Xiao, Y.; Yang, Q.; $\mathrm{Liu}, \mathrm{Y}$. One-step synthesis of amino-functionalized fluorescent carbon nanoparticles by hydrothermal carbonization of chitosan. Chem. Commun. 2012, 48, 380-382.

(18) Jaiswal, A.; Ghosh, S. S.; Chattopadhyay, A. One step synthesis of C-dots by microwave mediated caramelization of poly(ethylene glycol). Chem. Commun. 2012, 48, 407-409.

(19) Wang, W.; Li, Y.; Cheng, L.; Cao, Z.; Liu, W. Water-soluble and phosphorus-containing carbon dots with strong green fluorescence for cell labeling. J. Mater. Chem. B 2014, 2, 46-48.

(20) Yang, Y.; Wu, D.; Han, S.; Hu, P.; Liu, R. Bottom-up fabrication of photoluminescent carbon dots with uniform morphology via a soft-hard template approach. Chem. Commun. 2013, 49, 4920-4922.

(21) Zong, J.; Zhu, Y.; Yang, X.; Shen, J.; Li, C. Synthesis of photoluminescent carbogenic dots using mesoporous silica spheres as nanoreactors. Chem. Commun. 2011, 47, 764-766.

(22) Chandra, S.; Das, P.; Bag, S.; Laha, D.; Pramanik, P. Synthesis, functionalization and bioimaging applications of highly fluorescent carbon nanoparticles. Nanoscale 2011, 3, 1533-1540.

(23) Sarkar, S.; Das, K.; Ghosh, M.; Das, P. K. Amino acid functionalized blue and phosphorous-doped green fluorescent carbon dots as bioimaging probe. RSC Adv. 2015, 5, 65913-65921.

(24) Lim, S. Y.; Shen, W.; Gao, Z. Carbon quantum dots and their applications. Chem. Soc. Rev. 2015, 44, 362-381.

(25) Roy, P.; Chen, P.-C.; Periasamy, A. P.; Chen, Y.-N.; Chang, H.T. Photoluminescent carbon nanodots: synthesis, physicochemical properties and analytical applications. Mater. Today 2015, 18, 447458.

(26) Zhang, Y.; Wang, Y.; Feng, X.; Zhang, F.; Yang, Y.; Liu, X. Effect of reaction temperature on structure and fluorescence properties of nitrogen-doped carbon dots. Appl. Surf. Sci. 2016, 387, $1236-1246$.

(27) Song, Y.; Zhu, S.; Zhang, S.; Fu, Y.; Wang, L.; Zhao, X.; Yang, B. Investigation from chemical structure to photoluminescent mechanism: a type of carbon dots from the pyrolysis of citric acid and an amine. J. Mater. Chem. C 2015, 3, 5976-5984.

(28) Bhunia, S. K.; Pradhan, N.; Jana, N. R. Vitamin B1 derived blue and green fluorescent carbon nanoparticles for cell-imaging application. ACS Appl. Mater. Interfaces 2014, 6, 7672-7679.

(29) Hu, Y.; Yang, J.; Tian, J.; Yu, J.-S. How do nitrogen-doped carbon dots generate from molecular precursors? An investigation of the formation mechanism and a solution-based large-scale synthesis. J. Mater. Chem. B 2015, 3, 5608-5614.

(30) Zheng, B.; Liu, T.; Paau, M. C.; Wang, M.; Liu, Y.; Liu, L.; Wu, C.; Du, J.; Xiao, D.; Choi, M. M. F. One pot selective synthesis of water and organic soluble carbon dots with green fluorescence emission. RSC Adv. 2015, 5, 11667-11675.

(31) Li, Y.; Zhao, Y.; Cheng, H.; Hu, Y.; Shi, G.; Dai, L.; Qu, L. Nitrogen-doped graphene quantum dots with oxygen-rich functional groups. J. Am. Chem. Soc. 2012, 134, 15-18.

(32) Zhang, Y.-Q.; Ma, D.-K.; Zhuang, Y.; Zhang, X.; Chen, W.; Hong, L.-L.; Yan, Q.-X.; Yu, K.; Huang, S.-M. One-pot synthesis of Ndoped carbon dots with tunable luminescence properties. J. Mater. Chem. 2012, 22, 16714-16718.

(33) Zhou, J.; Shan, X.; Ma, J.; Gu, Y.; Qian, Z.; Chen, J.; Feng, H. Facile synthesis of $\mathrm{P}$-doped carbon quantum dots with highly efficient photoluminescence. RSC Adv. 2014, 4, 5465-5468.

(34) Chandra, S.; Patra, P.; Pathan, S. H.; Roy, S.; Mitra, S.; Layek, A.; Bhar, R.; Pramanik, P.; Goswami, A. Luminescent S-doped carbon dots: an emergent architecture for multimodal applications. J. Mater. Chem. B 2013, 1, 2375-2382.
(35) Ding, H.; Xiong, H.-M. Exploring the blue luminescence origin of nitrogen-doped carbon dots by controlling the water amount in synthesis. RSC Adv. 2015, 5, 66528-66533.

(36) Hou, J.; Wang, W.; Zhou, T.; Wang, B.; Li, H.; Ding, L. Synthesis and formation mechanistic investigation of nitrogen-doped carbon dots with high quantum yields and yellowish-green fluorescence. Nanoscale 2016, 8, 11185-11193.

(37) Yang, J.; Chen, W.; Liu, X.; Zhang, Y.; Bai, Y. Hydrothermal synthesis and photoluminescent mechanistic investigation of highly fluorescent nitrogen doped carbon dots from amino acids. Mater. Res. Bull. 2017, 89, 26-32.

(38) Papaioannou, N.; Marinovic, A.; Yoshizawa, N.; Goode, A. E.; Fay, M.; Khlobystov, A.; Titirici, M. M.; Sapelkin, A. Structure and solvents effects on the optical properties of sugar-derived carbon nanodots. Sci. Rep. 2018, 8, No. 6559.

(39) Liu, X.; Frank, B.; Zhang, W.; Cotter, T. P.; Schlogl, R.; Su, D. $\mathrm{S}$. Carbon-catalyzed oxidative dehydrogenation of $\mathrm{n}$-butane: selective site formation during sp3-to-sp2 lattice rearrangement. Angew. Chem., Int. Ed. 2011, 50, 3318-3322.

(40) Németh, P.; Garvie, L. A.; Buseck, P. R. Twinning of cubic diamond explains reported nanodiamond polymorphs. Sci. Rep. 2016, 5, No. 18381.

(41) Saxena, S.; Tyson, T. A.; Shukla, S.; Negusse, E.; Chen, H.; Bai, $\mathrm{J}$. Investigation of structural and electronic properties of graphene oxide. Appl. Phys. Lett. 2011, 99, No. 013104.

(42) Zhou, Z.; Zhang, H.; Zhou, Y.; Qiao, H.; Gurung, A.; Naderi, R.; Elbohy, H.; Smirnova, A. L.; Lu, H.; Chen, S.; Qiao, Q. Binder Free Hierarchical Mesoporous Carbon Foam for High Performance Lithium Ion Battery. Sci. Rep. 2017, 7, No. 1440.

(43) Xu, Y.; Wu, M.; Liu, Y.; Feng, X. Z.; Yin, X. B.; He, X. W.; Zhang, Y. K. Nitrogen-doped carbon dots: a facile and general preparation method, photoluminescence investigation, and imaging applications. Chemistry 2013, 19, 2276-2283.

(44) Ehrat, F.; Bhattacharyya, S.; Schneider, J.; Lof, A.; Wyrwich, R.; Rogach, A. L.; Stolarczyk, J. K.; Urban, A. S.; Feldmann, J. Tracking the Source of Carbon Dot Photoluminescence: Aromatic Domains versus Molecular Fluorophores. Nano Lett. 2017, 17, 7710-7716.

(45) Ferrari, A. C.; Robertson, J. Raman spectroscopy of amorphous, nanostructured, diamond-like carbon, and nanodiamond. Philos. Trans. R. Soc., A 2004, 362, 2477-2512.

(46) Ferrari, A. C.; Robertson, J. Interpretation of Raman spectra of disordered and amorphous carbon. Phys. Rev. B 2000, 61, 1409514107.

(47) Castiglioni, C.; Negri, F.; Rigolio, M.; Zerbi, G. Raman activation in disordered graphites of the $\mathrm{Al}^{\prime}$ symmetry forbidden $\mathrm{k} \neq 0$ phonon: The origin of the D line. J. Chem. Phys. 2001, 115, 37693778.

(48) Vidano, R. P.; Fischbach, D. B.; Willis, L. J.; Loehr, T. M. Observation of Raman band shifting with excitation wavelength for carbons and graphites. Solid State Commun. 1981, 39, 341-344.

(49) Campbell, I. H.; Fauchet, P. M. The effects of microcrystal size and shape on the one phonon Raman spectra of crystalline semiconductors. Solid State Commun. 1986, 58, 739-741.

(50) Ding, H.; Yu, S. B.; Wei, J. S.; Xiong, H. M. Full-Color LightEmitting Carbon Dots with a Surface-State-Controlled Luminescence Mechanism. ACS Nano 2016, 10, 484-491.

(51) Yang, W.; Zhang, H.; Lai, J.; Peng, X.; Hu, Y.; Gu, W.; Ye, L. Carbon dots with red-shifted photoluminescence by fluorine doping for optical bio-imaging. Carbon 2018, 128, 78-85.

(52) Sevilla, M.; Fuertes, A. B. Chemical and structural properties of carbonaceous products obtained by hydrothermal carbonization of saccharides. Chem. - Eur. J. 2009, 15, 4195-4203.

(53) Nie, H.; Li, M.; Li, Q.; Liang, S.; Tan, Y.; Sheng, L.; Shi, W.; Zhang, S. X.-A. Carbon Dots with Continuously Tunable Full-Color Emission and Their Application in Ratiometric $\mathrm{pH}$ Sensing. Chem. Mater. 2014, 26, 3104-3112.

(54) Xiong, Y.; Schneider, J.; Reckmeier, C. J.; Huang, H.; Kasak, P.; Rogach, A. L. Carbonization conditions influence the emission 
characteristics and the stability against photobleaching of nitrogen doped carbon dots. Nanoscale 2017, 9, 11730-11738.

(55) Wang, Y.; Kalytchuk, S.; Zhang, Y.; Shi, H.; Kershaw, S. V.; Rogach, A. L. Thickness-Dependent Full-Color Emission Tunability in a Flexible Carbon Dot Ionogel. J. Phys. Chem. Lett. 2014, 5, 14121420.

(56) Sudolská, M.; Dubecký, M.; Sarkar, S.; Reckmeier, C. J.; Zbořil, R.; Rogach, A. L.; Otyepka, M. Nature of Absorption Bands in Oxygen-Functionalized Graphitic Carbon Dots. J. Phys. Chem. C 2015, 119, 13369-13373.

(57) Kwon, W.; Do, S.; Kim, J.-H.; Seok Jeong, M.; Rhee, S.-W. Control of Photoluminescence of Carbon Nanodots via Surface Functionalization using Para-substituted Anilines. Sci. Rep. 2015, 5, No. 12604.

(58) Dubey, P.; Tripathi, K. M.; Mishra, R.; Bhati, A.; Singh, A.; Sonkar, S. K. A simple one-step hydrothermal route towards water solubilization of carbon quantum dots from soya-nuggets for imaging applications. RSC Adv. 2015, 5, 87528-87534.

(59) Deng, Y.; Chen, X.; Wang, F.; Zhang, X.; Zhao, D.; Shen, D. Environment-dependent photon emission from solid state carbon dots and its mechanism. Nanoscale 2014, 6, 10388-10393.

(60) Hu, X.; An, X.; Li, L. Easy synthesis of highly fluorescent carbon dots from albumin and their photoluminescent mechanism and biological imaging applications. Mater. Sci. Eng., C 2016, 58, 7306.

(61) Nguyen, V.; Si, J.; Yan, L.; Hou, X. Electron-hole recombination dynamics in carbon nanodots. Carbon 2015, 95, $659-663$.

(62) Zhang, R.; Liu, Y.; Yu, L.; Li, Z.; Sun, S. Preparation of highquality biocompatible carbon dots by extraction, with new thoughts on the luminescence mechanisms. Nanotechnology 2013, 24, No. 225601.

(63) Zhang, Y.; Wang, Y.; Guan, Y.; Feng, L. Uncovering the pKa dependent fluorescence quenching of carbon dots induced by chlorophenols. Nanoscale 2015, 7, 6348-6355.

(64) Williams, A. T. R.; Winfield, S. A.; Miller, J. N. Relative fluorescence quantum yields using a computer-controlled luminescence spectrometer. Analyst 1983, 108, 1067-1071.

(65) Fan, R.-J.; Sun, Q.; Zhang, L.; Zhang, Y.; Lu, A.-H. Photoluminescent carbon dots directly derived from polyethylene glycol and their application for cellular imaging. Carbon 2014, 71, 87-93.

(66) Shen, P.; Xia, Y. Synthesis-modification integration: one-step fabrication of boronic acid functionalized carbon dots for fluorescent blood sugar sensing. Anal. Chem. 2014, 86, 5323-5329.

(67) Wen, X.; Yu, P.; Toh, Y. R.; Ma, X.; Tang, J. On the upconversion fluorescence in carbon nanodots and graphene quantum dots. Chem. Commun. 2014, 50, 4703-4706.

(68) Dhami, S.; Mello, A. J. D.; Rumbles, G.; Bishop, S. M.; Phillips, D.; Beeby, A. Phthalocyanine Fluorescence at High Concentration: Dimers or Reabsorption Effect? Photochem. Photobiol. 1995, 61, 341346. 